\title{
La práctica de la investigación educativa desde la psicología: Intereses implícitos y finalidad social
}

\author{
The practice of educational research from psychology: \\ implied interest and social purpose
}

Claudia Carrasco Aguilar ${ }^{1}$

\section{Resumen}

El presente artículo busca desarrollar la tesis de que la Psicología y la Educación pueden vincularse en el ámbito de la Investigación Educativa, por medio de una relación que no ubique a ninguna de estas disciplinas en una posición de dominación sobre la otra. Tradicionalmente la Psicología se ha colocado al servicio de los intereses de reproducción social de la Educación, colaborando con su mantención por medio de estrategias de validación derivadas de las formas de investigación científica. Sin embargo, actualmente nos encontramos en un escenario en el cual las innovaciones y cuestionamientos éticos y políticos a estas formas de aproximarse al fenómeno educativo están comenzando a evidenciarse con mayor fuerza, demostrando que existen experiencias prácticas desde metodologías como la Sistematización de Experiencias y la Investigación-Acción, que colaboran con el cuestionamiento de la función social de la Investigación Educativa desde la intervención psicológica, con una finalidad última de emancipación social.

Palabras clave: Psicología, Investigación Educativa, Emancipación.

1 Escuela de Psicología, Pontificia Universidad Católica de Valparaíso. Chile. nadieseeducasolo@gmail.com 


\section{Abstract}

This article seeks to develop the thesis that the Psychology and Education can be linked in the field of Educational Research, by means of a relationship that does not fall into any of these disciplines in a relationship of domination on the other. Traditionally, the Psychology has been placed at the service of the interests of social reproduction of Education, working with its maintenance through strategies of validation arising from the forms of scientific research. However, we are currently at a scenario which the innovations and ethical questions and politicians to these forms of approaching phenomenon educational, are beginning to evident with greater force, demonstrating that there practical experiences from methodologies as the systematization of experiences and Research Action, who collaborate with the questioning of the social function of the Educational Research from the psychological intervention, with a purpose last social emancipation.

Key words: Psychology, Educational Research, Emancipation.

\section{Introducción y justificación del problema}

Durante una larga tradición de colaboración mutua la Psicología ha contribuido con la Educación desde diversos puntos de vista. Algunos elementos de cooperación han estado vinculados con una práctica de profundización de los componentes científicos a los que suele aspirar la Pedagogía, mientras que, al mismo tiempo, ha sido la Psicología la que ha otorgado herramientas técnicas que le permitan a la Educación reproducirse a sí misma como instrumento de mantención de las injusticias sociales. Esto último se ha evidenciado mayoritariamente en la validación de los supuestos epistemológicos y ontológicos de un ser humano carente, que la Psicología ha sostenido en su relación con la Educación, lo que se puede visualizar de forma especial cuando la Psicología interviene en el campo de investigación en Educación. Lo anterior se vincula con la forma en 
que muchas veces la Psicología se aproxima al fenómeno educativo como objeto de estudio, y las implicancias sociopolíticas que esta aproximación conlleva.

En reiteradas ocasiones se ha negado la necesaria inclusión de la dimensión ética en investigación educativa desde la Psicología, como si no fuese necesario un juicio de apreciación aplicado a la distinción entre el bien y el mal. Lo mismo ocurre con la dimensión política, en lo relativo a los derechos y deberes civiles y a las relaciones de poder y su dinámica (Montero, 2001). Para poder realizar un análisis de las prácticas de la investigación educativa desde la Psicología como disciplina es necesario aceptar que las propias ciencias sociales, y en especial la Psicología, deben girar hacia sí mismas las armas de la crítica, deconstruyendo los supuestos acríticamente asumidos que infiltran sus conceptualizaciones, teorías y procedimientos (Ibáñez, 1995).

Particularmente la Psicología se vuelve relevante y de interés cuando interviene en investigación educativa, porque termina volviéndose un cómplice de la ideología transmitida por la Educación (Bahía Bock, 2003) en cuanto esta última sería, en gran parte, responsable de la mantención de las desigualdades sociales en nuestro país.

Sin embargo, es necesario destacar que no siempre ni desde todas las aristas, la Psicología colabora con la reproducción de las desigualdades sociales. Existen corrientes en Investigación Educativa desde la propia Psicología que buscan precisamente materializar un vínculo que permita que ambas logren develar las implicancias sociales implícitas en esta colaboración, con el fin de transitar juntas hacia una meta común: la transformación social. En investigación social, en términos generales, siempre son los supuestos, intereses y propósitos los que llevan a un investigador a elegir una u otra metodología (Taylor \& Bogdan, 1992). Tradicionalmente, en ciencias sociales ha prevalecido fundamentalmente una tensión entre el positivismo, asociado a las metodologías cuantitativas; y la fenomenología, asociada a las metodologías cualitativas. Las primeras tienden a colabora con la mantención del status quo respecto del proyecto social, mientras que las segundas buscarían ofrecer una alternativa a lo anterior. 
Es por ello que en los últimos veinte años existe un auge de los métodos cualitativos, lo que se relacionaría con la emergencia de perspectivas críticas en investigación social (Iñiguez, 1999). Y es que la principal distinción entre los métodos cualitativos y cuantitativos no refiere a sus técnicas, sino al modelo conceptual a la base, es decir, a sus supuestos. Para Pérez Serrano (1994), con el fin de abordar en forma científica el problema metodológico, es necesario analizar el enfoque conceptual más adecuado para afrontar con éxito la investigación en el campo social, y problematizar en forma constante los supuestos e implicancias sociales, éticas y políticas a la base de una investigación. Para Ibáñez (1996), "Problematizar no es, solamente -sería demasiado fácil- conseguir que lo no problemático se torne problemático" (p. 54). Para este autor, problematizar implica comprender cómo y por qué algo ha adquirido un estatus de evidencia incuestionable. Es por esto que esos modos conceptuales dependerán, en definitiva, de los intereses que orientan las acciones a seguir.

A partir de lo anterior, se vuelve necesario explicitar el modelo conceptual a la base de las metodologías de investigación en educación, pero particularmente aquellas en que la Psicología ha sido su principal colaboradora. Para realizar este ejercicio es necesario apropiarse de los postulados de diversos autores, algunos de los cuales permiten visibilizar los elementos implícitos en las prácticas de investigación. Entre estos autores, Habermas (1990) se encarga de analizar cómo los diseños científicos y, por lo tanto, los modos de hacer ciencia, no son política ni ideológicamente neutrales, existiendo una clara relación entre conocimiento e interés, relación que estaría guiando el trabajo humano. En este sentido, el autor distingue entre el interés técnico, el interés práctico y el interés emancipatorio, que constituirían los tres tipos de ciencia mediante los que se genera y organiza el saber en nuestra sociedad. Estas tres formas de saber son la empírico-analítica, la histórico-hermenéutica y la crítica (Grundy, 1991).

Los planteamientos de Habermas se vuelven relevantes en cuanto posibilitan la construcción de un marco de entendimiento que favorezca el análisis de la metodología, como teoría que engloba las operaciones de 
una investigación (Bourdieu, Chamboredon \& Passeron, 2002). En este sentido, "el estudio crítico - y autocrítico-de las condiciones de producción del conocimiento en las ciencias sociales, ha sido uno de los intereses más continuos de Bourdieu" (Giglia, 2003, p. 151). Para Bourdieu et al., (2002) el concepto de reflexividad se vuelve crucial en estos análisis, ya que posibilitaría enfrentar las dicotomías derivadas de la dominación cultural. La reflexividad permitiría tomar distancia del sentido común, con el fin de comprender la generación de conocimientos como una forma de ruptura con lo real, la cual, a su vez, resultaría efectiva sólo si es que se genera un proceso constante de vigilancia para no recaer en el sentido común. A esto Bourdieu et al. (2002) llaman vigilancia epistemológica.

Desde los desarrollos teóricos de Habermas (1990), el ejercicio constante de la reflexividad de la que nos hablan Bourdieu et al. (2002) sería posible de ser llevado a cabo por medio de la ciencia crítica, producto, a su vez, del interés emancipatorio. En los planteamientos del autor, los intereses son constitutivos del conocimiento debido a que configuran y determinan lo que se considera objetos y tipos de conocimiento (Habermas, 1990). Por lo tanto, la forma que adquiera cada racionalidad determinará lo que un grupo social podrá distinguir como conocimiento, y por lo tanto, el interés por medio del cual se produzca este conocimiento determinará el cuerpo metodológico para investigar. En los términos de Bourdieu et al. (2002) esto es aún más complejo, ya que lo que se trataría de objetivar por medio de la reflexividad y la vigilancia epistemológica no es la experiencia vivida por el sujeto del conocimiento, sino más bien las condiciones de producción del conocimiento y las implicancias de las mismas. Para Giglia (2003):

“(...) la postura reflexiva se vincula con la adopción de una mirada relacional sobre los fenómenos, que por un lado, pone de manifiesto los objetos y sus contextos (los campos), y por el otro vincula el quehacer científico con su propio campo de producción, y de esa manera lo objetiva como producto histórico" (p. 153). 
Sin embargo, las relaciones entre Psicología y Educación, al momento de llevar a cabo procesos de investigación no suelen referirse a este aspecto como elemento de interés de sus prácticas. Para Bahía Bock (2003), la relación entre Psicología y Educación necesita ser analizada críticamente, develando el discurso y las concepciones construidas a partir de esta relación. Para la autora, la Psicología ha fortalecido las nociones naturalizadoras propias de la Pedagogía, y ha cooperado en el ocultamiento de lo que la educación tiene de proceso social, contribuyendo a lo que denomina una complicidad ideológica. Y es que, tal como manifiesta Montero (2001), la noción de paradigma en el marco de la investigación social ha sido entendida como un ámbito del saber configurado por las dimensiones ontológica, epistemológica y metodológica, obviando su carácter político. A partir de la teoría de los Intereses Constitutivos del Conocimiento de Habermas, es posible incorporar las dimensiones políticas e ideológicas de la Psicología en Investigación Educativa.

Lo anterior se vuelve relevante en cuanto una de las principales falencias de la Teoría Crítica en Educación ha sido la consecuencia práctica para seleccionar y organizar la cultura escolar hacia la transformación. Para Pinto (1997), al reclamar de estos planteamientos la ya mencionada consecuencia práctica “(...) emergen las insuficiencias teóricas de la propia opción práctica de la Teoría Crítica” (p. 6). Para el autor, estas insuficiencias estarían vinculadas con la incapacidad para superar la dualidad entre conciencia crítica y acción transformativa, así como una deficiencia para desarrollar una crítica al paradigma epistemológico y ontológico que constituye la racionalidad instrumental que es cuestionada.

En este sentido, cabe preguntarse ¿en qué medida la Psicología como disciplina colabora con la reproducción y/o transformación social al posicionarse en la Investigación Educativa desde intereses técnicos, prácticos y emancipadores? ¿De qué manera es posible conectar el discurso de la crítica con el discurso de la posibilidad en la Psicología cuando interviene en el campo de la Investigación Educativa? Siguiendo las ideas de Van Mannen (citado en Prieto, 2001a), el ejercicio autorreflexivo propio del interés emancipador se desarrolla cuando el individuo inicia procesos 
racionales críticos, lo que implicaría analizar las influencias ideológicas que actúan sobre la conciencia, con la finalidad de comenzar a liberarse de los dogmas dominantes que no facilitan el desarrollo de la identidad sino, más bien, la alienación y negación del sí mismo, y del entorno. En este mismo sentido, y siguiendo a Freire (1996), la realidad se construiría en relación con los intereses de los grupos que detentan el poder, pero también por todos quienes forman parte de la sociedad, otorgando así el carácter dialéctico propio de la Teoría Crítica y por consiguiente, del interés emancipador. Además, al tratarse de una construcción colectiva sería posible la transformación social.

Para dar respuesta a éstas y otras interrogantes, el presente artículo se apropia de los aportes de la Psicología Social Radical, la Psicología Social Crítica, así como de la Pedagogía Crítica. Para Iñiguez (2003) "La Psicología social radical enfatiza la transformación del orden social, es una psicología implicada en los procesos de emancipación y de cambio social" (p. 233), mientras que la Psicología social crítica:

\section{“(...) es sobre todo el resultado del continuo} cuestionamiento de las prácticas de producción de conocimiento. Puede ser radical o no, en el sentido de que puede permanecer al margen de cualquier pretensión de emancipación social o sentirse plenamente implicada en ella” (p. 233).

En este sentido, el análisis crítico al que se hace referencia se cuestiona por la relación de los fenómenos microsociales con las fuerzas sociales superiores, obedeciendo a ideales de justicia social, a la vez que busca desnaturalizar todo conocimiento construido, comprometiéndose con la emancipación y transformación social. Este nivel de análisis “(...) incorpora los elementos morales y éticos de la acción práctica involucrados en las distintas decisiones (...). Es decir, se reflexiona críticamente acerca de lo que sucede" (Prieto, 2001b, p.22). Para Pipper (2002), a una Psicología que conjugue los elementos críticos con los radicales, la convoca el deseo de alejarse del objetivismo cientificista y también del subjetivismo psicologicista, transitando un camino que es al mismo tiempo filosófico, 
práctico, político y ético. Estas formas de Psicología demuestran una disconformidad con la psicología convencional como profesión técnica y especialista en lograr diagnósticos y en intervenir eficazmente para transformar a los individuos. Para la autora, una Psicología Social Crítica y Radical opera por medio de la convicción de que existe una relación inmanente entre lo social y los procesos de construcción de significados, donde el lenguaje es la forma de acción social que los produce. Esta Psicología busca mantenerse ignorante de la división del conocimiento en disciplinas y subdisciplinas con especializaciones y subespecializaciones.

A partir de esto es que el entramado con la Pedagogía Crítica se vuelve relevante, toda vez que la Psicología busca intervenir en el campo de la Educación. La Pedagogía, siguiendo a Bazán, López y Yacometti (1995), implica una reflexión sistemática en torno a la Educación, al menos en dos dimensiones: una filosófica (asociada a una concepción sobre lo que es la educación, una concepción de la sociedad, y una concepción de los roles que les corresponde jugar al educador y al educando); y una científica, referida a la necesidad de abordar, explicar y comprender la Educación. Para esto, y siguiendo a los autores, la Pedagogía recurriría al aporte de varias ciencias de la educación como la Psicología Educacional, la Antropología Educacional, entre otras, pero también cuenta con disciplinas y saberes instrumentales propios como las teorías del Curriculum o Evaluación Educacional, por ejemplo. Uno de los problemas centrales en la actualidad para la Pedagogía sería el contexto social crítico donde los metarrelatos y los grandes ideales se habrían desvanecido. En este escenario, la Pedagogía necesitaría apuntar a un mundo mejor y más justo asumiendo al ser humano como sujeto social.

Es en el logro de la meta señalada recientemente que una interpretación restrictiva de la faceta psicológica podría determinar que la intervención pedagógica se centre exclusivamente en las diferencias de los sujetos y que se considere a éstas como única fuente de los problemas, prescindiendo de estudiar las restantes variables que influyen en el proceso educativo. De este modo, la pedagogía tradicional tendría una 
lectura de la realidad escolar altamente psicologizada, llevándola a ignorar frecuentemente la naturaleza histórica, social, cultural y política del hecho educativo y del sujeto que aprende (Bazán et al. 1995). Es por ello que la Pedagogía Crítica debe retomar los componentes éticos y políticos de los cuales ha tratado de ser desposeída por una Psicología Tradicional.

En este sentido, entonces, si se busca llevar a cabo un entrecruce entre la Psicología Social Crítica y Radical con la Pedagogía Crítica, será necesario estar atentos y atentas a lo que señala Bahía Bock (2003). Para la autora, la relación entre Psicología y Educación necesita ser analizada críticamente, develando el discurso y las concepciones construidas a partir de esta relación.

Respecto al papel de la Psicología como agente activo dentro de los procesos de Investigación Educativa, aparecen los planteamientos de Martín-Baró (1998), para quien sería posible plantear tres puntos esenciales para enfrentar lo que él llama “(...) el reto histórico para el cual probablemente no fuimos preparados” (p. 176). Estos puntos se vinculan con la idea de replantearse la imagen de sí mismo como profesional, asumir la perspectiva de las mayorías populares y realizar una confrontación crítica frente al sistema social. En síntesis, para este autor, “(...) se trata de poner el saber psicológico al servicio de la construcción de una sociedad donde el bienestar de los menos no se asiente sobre el malestar de los más" (p.177). De este modo, este análisis, desde las tres perspectivas señaladas, podría ser posible.

A continuación se realiza un análisis crítico con base en distintos desarrollos teóricos respecto de las relaciones sociohistóricas entre Psicología y Educación; las Teorías Críticas para su análisis; y las relaciones entre la Práctica Investigativa desde la Psicología en contextos educativos. Finalmente, se realiza una propuesta para materializar los análisis presentados, incluyendo, para ello, algunos ejemplos de procedimientos de investigaciones desde la Psicología en el campo educativo.

\section{Relaciones sociohistóricas entre Psicología y Educación}

Desde sus comienzos la Psicología en Educación se centra en el estudio de los procesos de aprendizaje y del desarrollo de la inteligencia 
de los sujetos escolarizados, aportando en la generación y desarrollo del conocimiento acerca del aprendizaje en general y respecto de lo que se espera se suscite a raíz de los procesos educativos. No obstante, estos aportes no han sido neutros en términos de las visiones, concepciones implícitas en ellos y en la construcción de mundo que han ido generando, como tampoco en sus efectos sobre las relaciones entre la Psicología y la Educación (Baltar, 2005). Por su parte, la inserción profesional de los psicólogos en la institución educacional se ha realizado principalmente a través del surgimiento de los servicios psicológicos escolares, derivándose en una conceptualización de la Psicología en Educación en términos más bien de Psicología Escolar (Baltar, 2003).

El quehacer del psicólogo designado por el sistema educacional chileno ha implicado una orientación mayoritariamente clínica y escasamente educativa, enfocándose en las dificultades de los estudiantes, realizando diagnósticos psicológicos y centrados más bien en la enseñanza que en el aprendizaje (López \& Yacometti, 1994 en Baltar, 2003). Estas prácticas en Psicología generan un efecto rotulador y estigmatizador de las acciones clínicas en la escuela (Baltar, 2003). Siguiendo los planteamientos de Baltar (2005), a partir de la década del cuarenta esta disciplina ha estado orientada al orden, predicción, gestión y control del medio educativo, reafirmando la tendencia de la escuela a la normalización. Luego, en la década del sesenta, emerge un fuerte cuestionamiento a la influencia del modelo médico en la Psicología en general, y en relación con la Educación en particular. Sin embargo, para Bahía Bock (2003) la Pedagogía y la Psicología que la acompaña en el trabajo educativo insisten en pensar la educación como un proceso natural de desarrollo de las potencialidades existentes en los sujetos, fortaleciendo las lecturas patologizantes que atribuyen la responsabilidad exclusivamente al educando y a su familia.

La Psicología dominante posee una concepción de ser humano que parte de la naturaleza humana, desarrollando prácticas de rehabilitación de los alumnos que se constituyen como problemas para la escuela. Es así como para Bahía Bock (2003) la complicidad ideológica entre Psicología y Educación coloca a la Psicología en el papel de proteger, por medio de la investigación en educación, lo que está establecido en la sociedad, operando 
por medio del pensamiento científico como autoridad para explicar lo que se quiere ocultar.

Para Bahía Bock, el principal problema de esta relación entre Psicología y Educación, mediatizada por la Investigación Educativa, se encuentra en que lo que se avala y mantiene son finalmente las desigualdades sociales, donde la principal contribución de la Psicología como disciplina ha estado en la responsabilización del individuo por su propio desarrollo, concibiendo su objeto de estudio como algo en sí mismo.

La contribución de las teorías críticas en el análisis de las relaciones sociohistóricas entre Psicología y Educación en el campo de la Investigación Educativa

A partir de la década del sesenta e inicios de los setenta, según Bonal (1998), comienza a aparecer una desconfianza en que el consenso y el equilibrio son los fundamentos de una sociedad normal, para comenzar a aceptar que el conflicto también es parte de la sociedad. Posteriormente surgen las Teorías Críticas que se nutren fundamentalmente de la sociología forjada a partir de la década de los ochenta. Para Pinto (1997) es necesario remitirse a los planteamientos de Habermas al hablar de Teoría Crítica, específicamente en lo vinculado con la intersubjetividad como constructora del conocimiento, la transitividad de la conciencia hacia la autonomía de la acción del sujeto, el enfoque dialéctico del interés transformativo de la realidad que adopta el sujeto sociocultural, y la comunicación como dimensión holística de la acción transformadora.

Para Habermas (1990) existe una relación intrínseca entre las formas de saber y los intereses que guían este conocimiento, por lo que no sería neutral tal y como ha sido proclamado por la ciencia moderna (Bazán, 2002). Asimismo, cada interés que guía la producción de nuevos conocimientos estaría relacionado con diversos valores y formas de concebir la educación (Carr, 1990). Para quienes interpretan la educación desde los planteamientos de Habermas, la finalidad de ésta debiera ser el desarrollo de una acción autónoma y responsable (Grundy, 1991). Para la autora, esto llevaría al desarrollo de prácticas de emancipación 
buscando una cierta independencia de todo aquello que se encuentra fuera del individuo, enfatizando el estado de autonomía y responsabilidad que esto implicaría. Asimismo, la emancipación sólo sería posible por medio de la autorreflexión, y se encontraría vinculada con las ideas de justicia e igualdad, por lo que la finalidad se orientaría hacia la búsqueda de la liberación de dependencias dogmáticas, donde se podría desarrollar la libertad. Carr (1990) coincide con lo anterior, al señalar que “(...) la aspiración de la aproximación crítica sería aumentar la autonomía racional de la comunidad educativa” (p. 83).

Carr y Kemmis (1988) plantean que para que esta autonomía se desarrolle sería necesario analizar no sólo las condiciones subjetivas que limitan la libertad humana, sino también las objetivas, y cómo podrían cambiar las unas y las otras. En este sentido, la Psicología puede intervenir en el campo de la Investigación Educativa contribuyendo al estudio de las condiciones de subjetividad subyugadas a determinadas estructuras de poder. Una vez que es posible asumir que ella no puede eliminar de sí los valores y la ideología, resulta imposible representarla como una simple “(...) búsqueda desinteresada de conocimientos” (Carr, 1990, p.134). Aún más, para el autor, “(...) la idea de que ella puede liberarse de valores y de ideología resulta ser una de las ilusiones ideológicas de la época” (p.134). Pese a que los valores e intenciones no se encuentren reconocidas y aparezcan en forma implícita, toda investigación siempre contendría una vinculación con los valores educativos (Carr, 1990), y, por lo tanto, con determinados intereses, destacando entre ellos el interés técnico, el práctico y el emancipatorio (Habermas, 1990).

El interés técnico opera por medio de una marcada orientación hacia el control del medio, cuyo saber se basa en la experiencia y la observación (Grundy, 1991). Según los planteamientos de Habermas (1990), esta forma de saber es conocida como positivismo. Para Pérez Serrano (1994), el paradigma naturalista del Positivismo en las ciencias sociales implicaría la consideración de la existencia de un mundo real, exterior e independiente de los individuos, y el conocimiento de ese mundo resultaría objetivo y externo. Este paradigma implicaría los supuestos de que el mundo social tendría existencia propia, independientemente de quien lo estudia (Prieto, 
2001b) conduciendo a una concepción instrumentalista del conocimiento y de la relación entre la teoría y la práctica (Usher \& Bryant, 1992). Para estos autores el paradigma positivista considera siempre al conocimiento como algo al margen de las prácticas sociales.

Esta corriente investigativa basada en el interés técnico sería favorecida por “(...) Psicólogos Educativos de una persuasión behavorista, teoría del currículo basada en objetivos, investigación educativa en el paradigma tradicional” (Carr, 1990, p. 78-79). En esta misma línea, Wiesenfeld (2000) realiza un cuestionamiento a los posicionamientos adoptados por el psicólogo como investigador a partir de este tipo de interés, en cuanto la despersonalización de los informes construidos sobre datos encontraría su fundamento bajo la justificación metodológica de que un mayor detalle afectaría la imagen de homogeneidad y, por ende, de potencial de generalización de los datos. Para Wiesenfeld (2000) esta relación de la Psicología como ciencia investigadora de los fenómenos educativos ubicaría al psicólogo en una actitud prepotente al suponer que puede “(...) hablar por el otro, e interpretar con precisión su mundo de vida (...)” (p.4).

Por otro lado, y en contraposición con lo anterior, nos encontramos frente al interés práctico, que sería considerado el interés por la comprensión (Habermas, 1990). En este caso, la producción de saber mediante el hecho de dar significado constituye la tarea asociada con las ciencias histórico-hermenéuticas (Grundy, 1991), y con el Paradigma Fenomenológico para el cual "(...) lo subjetivo no sólo puede ser fuente de conocimiento sino incluso presupuesto metodológico y objeto de la misma ciencia" (Pérez Serrano, 1994, p.19). Para la autora, hoy estaría fuera de toda duda que la complejidad de los fenómenos educativos no puede ser captada en toda su riqueza y profundidad a través del enfoque tradicional de carácter positivista. Prieto (2001b) sostiene que la realidad educativa implica procesos entre personas, no predecibles ni medibles, por lo tanto, bastante más complejos que el mundo natural. En este sentido, para Habermas (1990) el conocimiento hermenéutico siempre se encontrará mediado por la comprensión inicial del intérprete. En esta lógica aparece una tendencia cada vez más presente desde la Psicología en la Investigación Educativa. 
Ésta ha buscado, paulatinamente, lograr la comprensión de significados y prácticas de la comunidad educativa, contextualizando lo individual en la red de significados en la que los sujetos se encuentran inmersos (Banz, Garrido, Herrera \& Zamora, 2004).

Sin embargo, la práctica asociada a la investigación educativa desde este tipo de interés no se encuentra libre de contradicciones. Para Wiesenfeld (2000) es necesario considerar que es el investigador quien generalmente selecciona y se aproxima al contexto educativo, motivado por ciertos propósitos, buscando el acceso a la subjetividad de los distintos actores educativos, en una relación que no se da de forma simétrica. Del mismo modo, para la autora “(...) el/la investigador/a publica y gana prestigio y reconocimiento a partir de las vidas de otros sin que algo equivalente ocurra del lado del informante" (p.8). De este modo, se propician relaciones de poder, ocurriendo que en el establecimiento de la relación entre teoría y empiria el dato es expropiado y objetivado al lenguaje y conceptualizaciones del marco de referencia del investigador (Carr \& Kemmis, 1988), siendo éste un trabajo que se realiza sin considerar a los agentes involucrados.

Para Habermas (citado en Grundy, 1991), en este tipo de relaciones entre Psicología e Investigación Educativa nos encontraríamos frente a un falso consenso, sosteniendo finalmente la lógica del positivismo contra la cual paradojalmente se levanta el interés práctico.

Iniciando un camino de resistencia: propuestas para la Psicología en el campo de la Investigación Educativa

Para Habermas (1990), el interés emancipatorio se orientaría fundamentalmente por la búsqueda de la liberación de dependencias dogmáticas, apareciendo en esta línea el paradigma sociocrítico en Investigación Educativa, donde la investigación asume una perspectiva democrática del conocimiento, así como los procesos implicados en su elaboración. Al intervenir desde acá, la Psicología asume que la Educación está constituida por realidades situacionales de sujetos, por lo que se encuentra comprometida con la transformación de la realidad desde una dinámica liberadora 
y emancipadora de los sujetos implicados en ella (Pérez Serrano, 1994). Para Habermas (1990) esta consideración es crucial, en cuanto desde el interés emancipatorio los agentes educativos debiesen volverse participantes activos en los procesos de investigación, de modo tal que las relaciones de dominación con las que suele colaborar la Psicología según Bahía Bock (2003) dan paso a relaciones humanizantes.

En términos metodológicos aparece una serie de iniciativas de investigación educativa que colaboran con la operacionalización de lo anterior, algunas de las cuales se encuentran vinculadas con la tradición educativa, y otras con la propia Psicología. Desde las tradiciones más educacionales, aparece la Investigación Acción Colaboradora (Suárez, 2002; Elliot, 1990; Carr \& Kemmis, 1988), la Macro etnografía (Rockwell, 1980) y la Sistematización de Experiencias derivada de la Educación Popular en Latinoamérica (Barnechea, González \& Morgan, 1998; Jara, 2001, 2006; Martinica, 1999); mientras que desde los aportes de la Psicología Social Crítica y la Psicología Radical nos encontramos con el Análisis Crítico del Discurso (Iñiguez 2003; Ibáńez 1996; Martín Rojo \& Whitaker, 1998; Foucault, 1966), la Autoetnografía (Sixto, 2004; MonteroSieburth, 2006; Fernández, 2005), la Investigación Acción Participativa (Montenegro, 2001) y la Historia de Vida (Bertaux, 1993). Todas estas metodologías realizan una invitación problematizadora y participativa a la Psicología cuando ésta actúa en el campo de la Investigación Educativa. A continuación se señalan ciertas características de la Investigación Educativa desde el paradigma sociocrítico, y se analizan a partir de experiencias concretas de Investigación Educativa en las que participan psicólogos y psicólogas.

Asumir una visión global y dialéctica del fenómeno educativo a estudiar: algunas experiencias concretas

La tesis de Magíster en Investigación Educativa del Programa dictado por la Universidad de Humanismo $\mathrm{Cristiano}^{2}$, cuya autora es la

2 Llevó por nombre Construcción de racionalidades emancipatorias en estudiantes universitarios: Historias de sueños y luchas sociales. 
Psicóloga Mabel Bórquez González, y que contó con la participación de la autora de este artículo en calidad de colaboradora, estudió la construcción de racionalidades emancipatorias en estudiantes universitarios, desde una perspectiva dialéctica incorporando la vida del individuo y la vida social. Es así como la investigación se orientó a posibilitar la potenciación de la autonomía y responsabilidad de los sujetos participantes. Lo anterior, fue posible de ser realizado facilitando una producción de información que permitió el cuestionamiento, en forma individual y grupal, desde una perspectiva crítica, donde constantemente cada agente estableciera las relaciones entre conocimiento, sus prácticas y las fuerzas sociales más amplias.

Por su lado, la visión dialéctica del fenómeno educativo ha sido el elemento central a considerar en toda una línea de investigación vinculada al análisis de los procesos de construcción de identidad docente en el marco del Sistema de Incentivos y Evaluación Docente. El Centro de Investigación Avanzada en Educación (CIAE) es una iniciativa de la Universidad de Chile en conjunto con la Universidad de Concepción y la Pontificia Universidad Católica de Valparaíso, donde se llevó a cabo una investigación fundamentalmente liderada por psicólogos, la que finalizó el año $2008^{3}$, situada desde la Psicología Social con un énfasis en la Psicología Laboral Organizacional, construyendo análisis respecto de la identidad docente en el sector municipal, bajo el supuesto de que operan como cualquier trabajador público. Desde acá, la investigación concluye que las Políticas de Evaluación del Desempeño Docente implican nuevas versiones de identidad laboral que entrarían en conflicto con versiones de identidad laboral docente que históricamente habrían operado. En términos prácticos, se llevó a cabo un proceso que permitió que las prácticas discursivas, desde el Análisis Crítico del Discurso, fueran analizadas en virtud de los procesos de construcción de Subjetivación del docente en Chile. Bajo estas concepciones, se asume que la subjetividad se encuentra anclada a las condicionantes sociohistóricas, por lo que en estricto rigor no es la mirada de la identidad que tradicionalmente ha ofrecido la Psicología

3 Llevó por nombre Política de Evaluación e Incentivos al Desempeño Docente: Análisis de la dimensión social y subjetiva de la implementación que realizan sostenedores, directores y docentes. Sus autores fueron: Vicente Sisto Campos, Carmen Montecinos Sanhueza, Luis Ahumada Figueroa, Abelardo Castro Hidalgo, Cristián Bellei Carvacho. 
la que colabora con la comprensión del fenómeno, debiendo profundizar en el marco social y el propio devenir histórico que sitúa al docente en su actual definición de sí mismo. Esta forma de analizar la realidad, y por lo tanto, la forma de realizar la investigación, buscó rescatar la visión dialéctica entre lo individual y lo social. En términos procedimentales, esta mirada dialéctica opera en la forma de hacer las entrevistas, las que fueron hechas por medio de la técnica de entrevista activa-reflexiva (Denzin, 2001). Esta forma de entrevista constituye una interacción en la cual los participantes son asumidos como sujetos que interactúan abiertamente, donde el entrevistador se muestra como sujeto, poniendo sus ideas y juicios como opinión en el diálogo. La entrevista es considerada como una forma de acción social, y es en tanto copartícipe de la acción que logran aproximarse a los procesos en los cuales la realidad adquiere sentido de un modo cotidiano. En este encuentro conversacional se puede generar un contexto interpretativo de forma tal que las conexiones entre las prácticas de las cuales dará cuenta el entrevistado sean claras, y se vinculen en el mismo discurso con el contexto sociocultural.

El desarrollo de esta investigación motiva el diseño y realización de un estudio que avanza en analizar construcciones de identidad laboral en profesores del sistema municipal a partir de la implementación de las políticas de evaluación e incentivos al desempeño docente en Chile ${ }^{4}$. Este estudio posee tres fases, donde la tercera se centra fundamentalmente en el Análisis Crítico del Discurso, con base en el desarrollo de entrevistas activas-reflexivas (Denzin, 2001). De este modo, se busca dar continuidad a las prácticas investigativas, donde la psicología se aleje de su posición de experta por sobre los fenómenos educativos, asumiendo un lugar de cotidianidad lingüística, democratizando a la vez el propio proceso de investigación.

$4 \quad$ FONDECYT No 1090739 (2009-2012) denominado Identidades en disputa: construcciones de identidad laboral en profesores del sistema municipal a partir de la implementación de las políticas de evaluación e incentivos al desempeño docente en Chile. Sus autores son: Vicente Sisto Campos, Carmen Montecinos Sanhueza, Luis Ahumada Figueroa. 
Asumir una perspectiva democrática del conocimiento, asi como los procesos implicados en su elaboración

El estudio debe constituirse en una práctica participativa en la que tanto el investigador como los sujetos investigados comparten responsabilidades (Pérez Serrano, 1994). El Seminario de Grado para Optar al título de Psicólogo y Grado Académico de Licenciado en Psicología, de la Escuela de Psicología de la Universidad de Valparaíso, llevado a cabo por Dennis Jensen y Cristian Villegas el año 2009, y que contó con la participación de la autora de este artículo en calidad de Asesora Metodológica, tuvo como tema una Sistematización de Experiencias del proceso de asesoramiento educativo en el marco del Programa Liceos Prioritarios. La democratización del proceso de construcción de conocimiento estuvo dada por la constitución de un equipo investigador con cuatro representantes del equipo de asesores, con quienes diseñaron el proyecto de sistematización, mientras que el análisis y la construcción de categorías fue realizado en conjunto con el equipo asesor completo, por medio de diversas técnicas participativas de investigación, dando cuenta así de la democratización del conocimiento construido.

En términos generales, las sistematizaciones de experiencias, como investigaciones con una fuerte tradición desde la Educación Popular, son las que mayormente dan cuenta de este principio de investigación. "La sistematización de experiencias es una metodología de investigación participativa iniciada por colectivos comprometidos con la Educación Popular en América Latina” (Verger, 2002, p.2), la que surge desde una ruptura paradigmática debido a los quiebres producidos por los movimientos sociales y políticos en reacción al contexto de crisis socioeconómica en la mayoría de los países de América Latina en las décadas de los setenta y ochenta (Jara, 2006).

La experiencia latinoamericana tiene mucho que decir al respecto, tal como señala Morgan (1996) al referirse al Taller Permanente de Sistematización (TPS). Éste surge el año 1988, al realizarse en Lima un Encuentro Nacional de las ONG afiliadas al Consejo de Educación de Adultos de América Latina (CEAAL). Allí se convocó a las instituciones y personas interesadas en la sistematización de experiencias de educación 
de adultos y promoción del desarrollo, a una jornada de trabajo sobre el tema. En Perú, una de las integrantes del Taller (Barnechea, 1992, citada en Morgan, 1996) inició la sistematización de su experiencia de promoción con comedores populares en un distrito de Lima. Para Morgan (1996) el problema más importante cuando estas formas de investigación tratan de asumir una perspectiva democrática del proceso, aparece en el análisis de la información. Y es que para la autora este momento exige un esfuerzo mental que los prácticos no estarían acostumbrados a realizar de manera explícita y consciente. Ésta es una de las tensiones más difíciles de resolver en Psicología, ya que su historia la empuja a imponer formas de categorización de la realidad por sobre las formas de explicación que un grupo de educadores puede ofrecer en términos metodológicos, pasando a llevar uno de los principios clave en la investigación sociocrítica: la participación. Para Jara (2006) un criterio común que puede ayudar a resolver esta tensión sería considerar que los actores no son meros informantes, y que los sujetos de la experiencia educativa pueden participar en su sistematización aportando en la interpretación crítica y no sólo dando informaciones para que otra persona las interprete.

En esta línea, y destacando la participación como forma de desarrollar conciencia crítica, aparece la Investigación Acción. Siguiendo los planteamientos de Carr y Kemmis (1988), la Investigación-Acción sería, en su esencia, una forma de indagación autorreflexiva que emprenderían los participantes en situaciones sociales con el fin de mejorar la racionalidad y la justicia de sus propias prácticas, su entendimiento de las mismas y las situaciones dentro de las cuales ellas tienen lugar. Lo interesante de esta línea es que es la Educación, y especialmente la Educación Popular en Latinoamérica, la que ha ido influyendo sobre la Psicología en las formas de organizar y de llevar a cabo los procesos de investigación. Es acá donde, en el contexto educativo formal, se comienza a evidenciar un cruce entre la Psicología Comunitaria y la Psicología Educacional, toda vez que esta última comienza a investigar con base en las herramientas metodológicas que tradicionalmente fueron de uso de la primera (Aceituno, Alfaro, Asún, Krause \& Morales, 1995). Un ejemplo de esto han sido las iniciativas lle- 
vadas a cabo en los proyectos implementados por GALERNA, que es una Plataforma de Trabajo enfocada al Desarrollo Social. Esta organización opera con un equipo de Consultores Profesionales desde el año 2002 a la fecha. Entre 2006 y 2008 se realizó un proyecto de investigación financiado por la División de Seguridad Pública del Ministerio del Interior, denominado "Prevención de la violencia y promoción de conductas prosociales en establecimientos educacionales”. En el marco de este proyecto, se desarrolló una estrategia metodológica de investigación- acción, a través de diagnósticos participativos, trabajo colaborativo, fortalecimiento de la organización de estudiantes a través de talleres de formación de líderes, conformando grupos que reflejaran la diversidad de los y las estudiantes, materializando una forma de mutua colaboración y participación en el trabajo de psicólogos investigadores en el contexto educativo.

\section{Comentarios finales}

La Psicología posee un pasado que la ha ubicado en distintos momentos desde una posición de poder, la que se ve reafirmada en las formas en que ha participado junto a la Investigación Educativa. Desde un interés técnico, el énfasis se ubica en el control y predicción, y la gestión del mundo se materializa en el positivismo, posicionando al paradigma naturalista como despojado de ideología, destacándose por una supuesta neutralidad. La Psicología colabora desde acá, con la rotulación de los estudiantes, con la normalización y homogenización de los agentes y procesos educativos, y con la mantención de un orden social injusto. En contraposición con esto, desde un interés práctico el énfasis se ubica en la comprensión, materializada en la hermenéutica como instrumento interpretativo. Desde el ámbito de la Psicología en Educación se tornan representativos de esta mirada los enfoques que relevan el papel del sujeto en los procesos de construcción social. Sin embargo, en el ámbito de la Investigación en Educación, la Psicología suele caer en las relaciones de poder indicadas como propias del interés técnico, en cuanto frecuentemente es el psicólogo investigador en Educación quien elabora los informes de investigación al margen de los agentes educativos que participan en dichos procesos, instrumentalizando 
los aspectos metodológicos asociados al interés práctico por medio de fines que obedecen más bien al interés técnico. A partir de esto, se proponen metodologías que asuman el supuesto de la participación activa en los procesos investigativos, donde el objeto de la Investigación Educativa llevada a cabo por la Psicología no sólo se encuentre concentrado en la promoción de la salud en la escuela, sino que considere las dos dimensiones centrales que destaca Bahía Bock (2003): la ética y la política.

De este modo se puede facilitar que el ser humano sea pensado como ser histórico y social, que actúa de manera transformadora sobre el mundo y se transforma también. Para la autora, esto podría posibilitar que la Psicología llegue a contribuir con que el educador comprenda tanto la importancia de la planificación de las situaciones educativas como la de enriquecer la enseñanza con contenidos de la realidad próxima de los educandos, ya que todos estos elementos serían condiciones para la construcción de un mundo psicológico saludable, en la medida que posibilitan al estudiante ampliar su comprensión del mundo que lo circunda, potenciando su intervención transformadora sobre la realidad cotidiana. Es hacia acá, de la mano de metodologías como la Sistematización de Experiencias y la Investigación Acción, hacia donde debiera orientarse la investigación psicológica en el área de la educación.

\section{Referencias}

Asún, D., Krause, M., Aceituno, R., Alfaro, J. \& Morales, G. (1995). La psicología comunitaria en Chile. Análisis de sus características y perspectivas. En E. Wiesenfeld \& E. Sánchez (Eds.). Psicología Social Comunitaria. Contribuciones latinoamericanas. (Pp. 115-178). Caracas: Tropikos.

Bahía Bock, A. (2003). Psicología de la Educación: Complicidad Ideológica. En A. Bahía Bock, A. Checcia \& M. Rebello De Souza (Eds.). Psicologia Escolar: Teorias críticas.(Pp. 79-103). Sao Paulo: Casa do Psicólogo.

Baltar, M. (2003). El sentido del diagnóstico psicológico escolar. Un análisis crítico y una propuesta en construcción. Revista Psicoperspectivas, 2, 7-34. 
Baltar, M. (2005). Estudio cualitativo de los aprendizajes en la etapa de formación inicial en el área de la psicología educacional a través de experiencias de trabajos en terreno; una mirada desde el discurso de los estudiantes de dos instituciones formadoras de psicólogos. Tesis de Magíster no publicada. Pontificia Universidad Católica de Valparaíso. Viña del Mar, Chile.

Banz, C., Garrido, A., Herrera, P. \& Zamora, A. (2004). Apunte de Reflexión: Bases para la Construcción de una Psicología Educacionalcomunitaria. Universidad Academia de Humanismo Cristiano, Escuela de Psicología. Santiago, Chile. Recuperado en abril, 2006: http://psicologia.academia.cl/apunte_reflexion.doc

Barnechea, M., González, E. \& Morgan, M. (1998). Sistematización de prácticas de animación sociocultural y participación ciudadana en América Latina. Ponencia presentada al Seminario Latinoamericano: Medellín, Fundación Universitaria Luis Amigó-CEAAL. Recuperado en junio, 2008. http://www.alforja.or.cr/sistem/Morgan.doc

Bazán, D., López, A. \& Yacometti, O. (1995). Una mirada prospectiva de la Psicopedagogía, En R. Careaga. (Ed.). Desafíos y Dilemas de la Psicopedagogía. (Pp. 58-70). Santiago: Bravo y Allende Editores.

Bazán, D. (2002). Pedagogía social y pedagogía crítica: nexos y fundamentos básicos. Revista de Pedagogía Crítica Paulo Freire, 1, 42-55.

Bertaux, D. (1993). De la perspectiva de la historia de vida a la transformación de la práctica sociológica. En C. Santamarina \& J. Marinas (Eds.). La historia oral: Métodos y experiencias. (Pp. 19-34). Madrid: Debate.

Bonal, X. (1998). Sociología de la educación. Una aproximación crítica a las corrientes contemporáneas. Barcelona: Paidós.

Bourdieu, P., Chamboredon, J.C. \& Passeron, J.C. (2002). El oficio de sociólogo. México: Siglo XXI.

Bórquez, M. (2007). Construcción de racionalidades emancipatorias en estudiantes universitarios: Historias de sueños y luchas sociales. Tesis de Magíster no publicada. Universidad Academia de Humanismo Cristiano, Santiago, Chile.

Carr, W. \& Kemmis, S. (1988) Teoría crítica de la enseñanza. Buenos Aires: Martínez Roca.

Carr, W. (1990). Hacia una ciencia crítica de la educación. Barcelona: Laertes.

Denzin, N. (2001). The reflexive interview and a performative social science. Qualitative Research, 1(1), 23-46.

Elliot, J. (1990). La investigación-acción en educación. Madrid: Morata. 
Fernández, R. (2005). Análisis de discurso como método autoetnográfico: Una experiencia en las conmemoraciones del 11 de septiembre. Ponencia presentada en el VI Congreso Latinoamericano de Estudios del Discurso. América Latina en su discurso. Centro de extensión PUC. Recuperado en agosto, 2006: http://www.congresoaled2005.puc.cl/ pdf/fernandez\%20roberto.pdf

Foucault, M. (1966). La arqueología del saber. Madrid: Siglo XXI.

Freire, P. (1996). Pedagogía de la esperanza. México: Siglo XXI.

Giglia, A. (2003). Pierre Bourdieu y la perspectiva reflexiva en las Ciencias Sociales. Rev. Desacatos, 11, 149-160.

Grundy, S. (1991). Producto o praxis del curriculum. Madrid: Ediciones Morata.

Habermas, J. (1990). Conocimiento e interés. Madrid: Taurus.

Ibáñez, T. (1995). La tensión esencial de la Psicología social. Prólogo a Páez, D. Valencia, J., Morales, J., Sarabia, B., \& Ursúa, N. (1995) Teoría y método en Psicología Social. Barcelona: Antrophos.

Ibáñez, T. (1996). Fluctuaciones conceptuales en torno a la postmodernidady la psicología. Caracas: Universidad Central de Venezuela.

Iñiguez, L. (1999). Investigación y evaluación cualitativa: bases teóricas y conceptuales. Revista Atención Primaria, 23(8), 496 - 502.

Ińiguez, L. (2003). Análisis del discurso. Manual para las ciencias sociales. Barcelona: Editorial UOC.

Jara, O. (2001). Dilemas y Desafíos de la Sistematización de Experiencias. Ponencia presentada en Seminario ASOCAM: Agricultura Sostenible Campesina de Montaña. Cochabamba, Bolivia. Recuperado en abril, 2009: http://www.grupochorlavi.org/webchorlavi/sistematizacion/ oscarjara.PDF

Jara, O. (2006). Sistematización de Experiencias y Corrientes Innovadoras del Pensamiento Latinoamericano. Una aproximación Histórica. La Piragua, Revista Latinoamericana de Educación y Politica, 23, 7-16. CEAAL. Recuperado en abril, 2009: http://ceaal.org/images/ documentos/lapiragua23-1.pdf

Jensen, D. \& Villegas, C. (2009). Sistematización de la experiencia de elaboración del modelo de trabajo, desarrollado por el equipo de asesoría técnica de la Escuela de Psicología de la Universidad de Valparaiso, al Programa Liceos Prioritarios - Mineduc. Seminario de grado y título no publicado. Escuela de Psicología, Facultad de Medicina, Universidad de Valparaíso.

Martín-Baro, I. (1998). Psicología de la liberación. Madrid: Trotta.

Martín Rojo, L. \& Wittaker, R. (1998). Poder-decir o el poder de los Discursos. Madrid: Arrecife. 
Martinic, S. (1999). El objeto de la sistematización y sus relaciones con la evaluación y la investigación. La Piragua, Revista Latinoamericana de Educación y Politica, 16, 44-51. Recuperado en abril, 2009: http://ceaal.org/images/stories/La\%20Piragua\%2016\%20 Sistematizacion\%20de\%20Practicas\%20en\%20L.pdf

Montenegro, M. (2001). Conocimientos, Agentes y Articulaciones: una mirada situada a la intervención social. Tesis doctoral no publicada. Universitat Autónoma de Barcelona.

Montero, M. (2001). Ética y política en Psicología. Las dimensiones no reconocidas en Athenea Digital, O. Recuperado en junio 2007: http://antalya.uab.es/athenea/num0/maritza.htm

Montero-Sieburth, M. (2006). La Auto-etnografía como una estrategia para la transformación de la homogeneidad a favor de la diversidad individual en la escuela. Universidad de Massachusetts-Boston. Ponencia Presentada en Congreso Nacional de Educación Intercultural Formación del Profesorado y Práctica Escolar, España. Recuperado en marzo 2007: http://www.uned.es/congreso-intereducacion-intercultural/

Morgan, M. (1996) Taller Permanente de Sistematización: búsquedas teóricas y epistemológicas desde la práctica de la sistematización. En E. Santibáñez y C. Âlvarez (Eds.). Sistematización y producción de conocimientos para la acción. (Pp. 40-48). Santiago: CIDE.

Piper, I. (2002). Sobre una práctica que, en el sur, se llama a sí misma psicología social crítica. En I. Piper, (2002) (Comp.). Políticas, Sujetos y Resistencias: Debates y Críticas en Psicología Social. Cuadernos de Psicología Social, 1, 19-33.

Pérez Serrano, G. (1994). Investigación Cualitativa. Retos e Interrogantes. Madrid: La Muralla.

Prieto, M. (2001a). Mejorando la Calidad de la Educación: Hacia una Resignificación de la Escuela. Valparaíso: Ediciones Universitarias de Valparaíso de la Universidad Católica de Valparaíso.

Prieto, M. (2001b). La investigación en el aula ¿una tarea posible? Valparaíso: Ediciones Universitarias de Valparaíso de la Universidad Católica de Valparaíso.

Rockwell, E. (1980). La relación entre etnografía y teoria de la investigación educativa. Ciudad de México: DIE, Centro de Estudios Avanzados.

Pinto, R. (1997). Una mirada crítica a los fundamentos históricos y situacionales de la educación chilena, en los años 90. Revista Docencia, 3, 5-13.

Sisto, V. (2004). Teoria(s) Organizacional(es) Postmoderna(s) y la Gest(ac) ión del Sujeto Postmoderno. Tesis doctoral no publicada. Universitat Autónoma de Barcelona. 
Suárez, M. (2002). Algunas reflexiones sobre la investigación-acción colaboradora en la educación. Revista Electrónica de Enseñanza de las Ciencias, 1(1), 40-56. Recuperado en marzo 2006: http://reec.uvigo. es/volumenes/volumen1/Numero1/Art3.pdf

Taylor, S.J. \& Bogdan, R. (1992) Introducción a los métodos cualitativos de investigación. Barcelona: Paidós.

Usher, R. \& Bryant, I. (1992). La educación de adultos como teoría, práctica e investigación. El triángulo cautivo. Madrid: Morata.

Verger, A. (2002). Sistematización de Experiencias en América Latina. Una Propuesta para el Análisis y la Recreación de la Acción Colectiva desde los Movimientos Sociales. Dpto. de Sociología de la Universitat Autónoma de Barcelona. Recuperado en marzo, 2006: http://www. alforja.or.cr/sistem/sistemat_verger.pdf

Wiesenfeld, E. (2000). Entre la prescripción y la acción: la brecha entre la teoría y la práctica en las investigaciones cualitativas. Forum Qualitative Social Research (On line Journal), 1(2). Recuperado en mayo 2007: http://qualitative-research.net/fqs/fqs-e/2-00inhalt-e. htm

Fecha de recepción: 29 de abril de 2010

Fecha de aceptación: 20 de agosto de 2010 